\title{
Doctors conflicted about patients recording clinical visits
}

\author{
Cite as: CMAJ 2018 November 5;190:E1316. doi: 10.1503/cmaj.109-5675
}

Posted on cmajnews.com on Oct. 18, 2018.

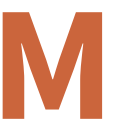

any doctors are uncomfortable that an increasing number of patients are recording clinical visits, although some physicians encourage the practice. A recent Dartmouth Institute study found that nearly one in five patients had recorded a clinical visit, and more than three in five were interested in doing so in the future. Men, younger people and those who spoke languages other than English at home were most likely to make recordings.

Some doctors are embracing the trend; $28 \%$ reported making recordings of visits for their patients. According to study lead Paul Barr, "we know that up to $80 \%$ of health care information is forgotten by patients after their clinic visit." Studies also show that "access to recordings can improve patient satisfaction and increase understanding of medical information."

However, doctors who hadn't made recordings for patients were split on whether they were willing to do so. Doctors' attitudes about taping visits also varied by specialty. Those in oncology and physical rehabilitation were most likely to have recorded visits for patients; family doctors were least likely. Only 1 in

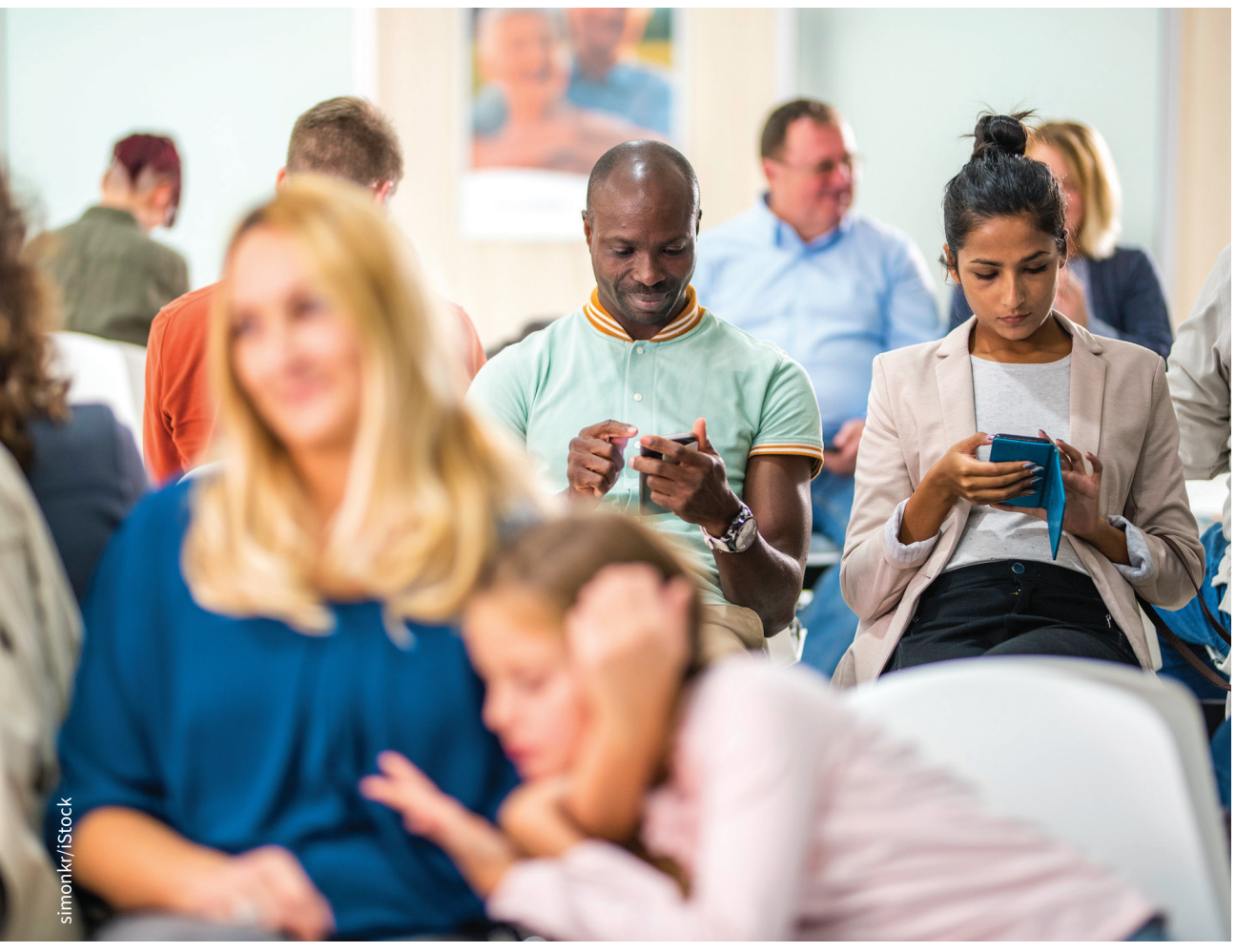

Nearly one in five patients record visits with their doctors, found an American study.
10 patients said their doctors' offices offered recordings for personal use.

Some clinicians may worry that allowing recordings will invite trouble. Physician requests for legal assistance with patient complaints have increased $85 \%$ over the past decade, according to the Canadian Medical Protective Association (CMPA). Misunderstandings related to recordings can lead to complaints, CMPA warns. Doctors can also be on the hook for breach of privacy if a recording captures identifiable information about other patients or staff.

The CMPA advises doctors to set policies and make agreements with patients in advance about whether and where recordings are allowed. If patients request to tape a visit, doctors should find out why, offer alternative solutions and use discretion in continuing the visit if the patient won't take no for an answer.

Fewer than 1 in 20 patients reported recording clinical visits without physician permission, but nearly 1 in 10 said they would consider doing so in future, according to the Dartmouth Institute study.

Barr and the other researchers argued that policy guidance for both doctors and patients is "urgently required." Of the 49 health systems they surveyed, only 2 had a policy on requests for audio and video recordings of clinical visits. None had policies or guidance for doctors or patients on the practice of sharing recordings.

"US clinicians and the public are taking the lead on sharing clinic visit recordings, while policy makers lag behind," the study concluded.

Lauren Vogel, CMAJ 